\title{
Role reversal: infiltrating T cells protect the brain
}

\author{
Lawrence Steinman
}

Departments of Pediatrics and Neurology and Neurological Sciences, Stanford University, Stanford, California, USA

\begin{abstract}
Inflammatory conditions intensify and then resolve, often sparing and recovering some of the injured tissue. While the ebb and flow of inflammation can be followed in many tissues, there is not a great deal of information on how inflammation regresses in the brain. In this issue of the $J C I$, Walsh, Hendrix, and colleagues illuminate a cellular mechanism whereby T cells that infiltrate the brain after nerve crush or contusion actually protect neurons from injury. These infiltrating T cells produce IL-4 and do so independently of a classic adaptive $T$ cell immune response. The T cells respond to mediators produced by damaged neurons, without the classic three-way interaction among antigen, the major histocompatibility complex, and the $T$ cell receptor. After brain injury, these protective $T$ cells produce IL-4, which attenuates damage via IL-4 receptors on neurons.
\end{abstract}

genic, altered MBP protects spinal cord neurons in a contusion model (6). These early studies focused on traditional $\mathrm{T}$ cells that recognize specific antigens and participate in the classic trimolecular interaction among TCR, MHC, and antigen.

In this issue, Walsh, Hendrix, and colleagues describe a previously unappreciated aspect of beneficial autoimmunity in the nervous system and identify a population of $\mathrm{T}$ cells that infiltrate the CNS after traumatic injury and provide protection that is independent of MHC presentation of antigen (9). This protective T cell population produced IL-4, which in turn mediated protective effects though interactions with neuronal IL-4 receptors.

\section{Protective immunity in brain inflammation}

Injury to the nervous system comes in many forms. In models of brain trauma, ischemia, autoimmunity, and infection, a traditional cellular infiltration of macrophages, T cells, B cells, and even granulocytes is observed. Classic autoimmune pathology in the brain is best studied in the now 82-year-old experimental autoimmune encephalomyelitis (EAE) model $(1,2)$. There are also numerous models of infectious damage to the brain, such as meningitis and encephalitis induced by bacteria and viruses $(3,4)$. Moreover, neurodegenerative conditions, such as Alzheimer's disease, that are characterized by amyloid deposits proceed without any signs of the classic inflammatory reaction seen in trauma, ischemia, EAE, or viral encephalitis (5). In models of ischemic and traumatic damage to the brain and in EAE as well as viral encephalitis, a resolution of the initial damage is seen, and this resolution often correlates with neurologic recovery (1-4).
The damage-induced cellular infiltrate that emanates from outside the nervous system contains constituents that provide protection from injury. The concept of beneficial autoimmunity was first described in a 1999 study that showed that injection of myelin basic protein-recognizing (MBP-recognizing) $\mathrm{T}$ cells insulates axons, allowing for recovery from a nerve crush injury of the optic nerve (6). In this system, there was a three-fold increase in retinal ganglion cells following administration of MBP-recognizing $\mathrm{T}$ cells as the result of a classic interaction between the $\mathrm{T}$ cell receptor and a MBP fragment presented by MHC class II protein. This initial demonstration of protective autoimmunity in the nervous system has been followed by studies that have further refined our understanding of this process. Engineered T cells that recognized an altered form of $\mathrm{MBP}$ are not capable of producing encephalomyelitis themselves but instead produce antiinflammatory cytokines, such as IL-4 $(7,8)$, that mediate neural protection. Injection of $\mathrm{T}$ cells that are sensitized to the nonpatho-

\section{Related Article: p. 699}

Conflict of interest: Lawrence Steinman has ownership in Atreca and Tolerion; receives income from Atreca, Raptor Pharmaceutical Corp., and Teva Pharmaceutical Industries; and received research support from Pfizer, Biogen Idec, and JT International.

Reference information: / Clin Invest. 2015;125(2):493-494. doi:10.1172/JCl80279.

\section{Protective T cells in the nervous system: mechanistic insights}

What signal generated by the damaged neurons triggered infiltrating $\mathrm{T}$ cells to produce IL-4? To answer this question, Walsh, Hendrix, and colleagues searched meticulously for a soluble factor that serves as a signal that neurons were "damaged" (9). The production of such an "alarmin" was mediated in part by MyD88, which transmits signals for many TLRs of the innate immune system. Specifically, T cells from MyD88-deficient mice exhibited reduced IL-4 production, and protection was impaired in these animals. Walsh, Hendrix, and colleagues did not find that any known TLR ligands mediate this protection. Likely candidates for the secreted factor that is involved in the MyD88-linked protection include members of the IL-1 family of cytokines, including IL- $1 \alpha$ and IL-1 $1 \beta$ as well as IL-18 and/or IL-33. The next step in this exciting story will likely be the identification of the precise alarmin that triggers the protective reaction in response to CNS damage (9).

What do the results of Walsh, Hendrix, and colleagues imply about current strategies used to treat the degenerative phase of inflammatory diseases like EAE and multiple sclerosis (MS)? Current approaches, including blockade of homing molecules like $\alpha 4$ integrin (10-12) and modulation 
of the sphingosine phosphate receptor in lymph nodes $(13,14)$, are designed to limit migration of lymphocytes into the CNS. If migration or function of $\mathrm{T}$ cells, which produce protective cytokines, is blocked by such therapies, the potential of the strategies to provide benefit during the neurodegenerative phases of diseases like MS may be impaired. For example, a recent clinical trial of fingolimod, which modulates the S1P receptor, showed that this strategy is ineffective for primary progressive MS (15). As studies on the immune response in the CNS expand, there is increasing evidence that the $\mathrm{T}$ cells that infiltrate the site of injury play dual roles. Some of these T cell populations cause harm and exacerbate damage, while others, such as the IL-4-producing population identified by Walsh, Hendrix, and colleagues, may provide benefit. The development of selective therapies that aim to eliminate harmful $\mathrm{T}$ cells while preserving and/or promoting $\mathrm{T}$ cells that provide protection should be explored. The study by Walsh, Hendrix, and colleagues is exciting and promising, as their identification of a $\mathrm{T}$ cell population that produces the protective cytokine IL-4 without classic recognition of neural antigens via the MHC provides a population to target for future therapeutic strategies aimed at limiting CNS damage.

\section{Acknowledgments}

I thank the NIH and the National MS Society for continued support over the past 40 years. I also thank various benefactors, including Sally and John Endriz and the Phil N. Allen Trust, who have supported research in my laboratory.

Address correspondence to: Lawrence Steinman, Beckman Center for Molecular Medicine, B002 279 Campus Drive, Stanford University, Stanford, California 94305, USA. Phone: 650.725.6401; E-mail: steinman@stanford.edu.

1. Rivers TM, Sprunt DH, Berry GP. Observations on attempts to produce acute disseminated encephalomyelitis in monkeys. JExp Med. 1933;58(1):39-53.

2. Steinman L, Zamvil SS. How to successfully apply animal studies in experimental allergic encephalomyelitis to research on multiple sclerosis. Ann Neurol. 2006;60(1):12-21.

3. Kulcsar KA, et al. Interleukin 10 modulation of pathogenic Th17 cells during fatal alphavirus encephalomyelitis. Proc Natl Acad Sci U S A. 2014;111(45):16053-16058.

4. Garraud K, et al. Differential role of the interleukin-17 axis and neutrophils in resolution of inhalational anthrax. Infect Immun. 2011;80 (1):131-142.

5. Steinman L. Nuanced roles of cytokines in three major human brain disorders. J Clin Invest. 2008;118(11):3557-3563.

6. Moalem G, et al. Autoimmune T cells protect neurons from secondary degeneration after central nervous system axotomy. Nat Med. 1999;5(1):49-55.

7. Brocke $S$, et al. Treatment of experimental encephalomyelitis with a peptide analogue of myelin basic protein. Nature. 1996;379(6563):343-346.

8. Hauben E, et al. Posttraumatic therapeutic vaccination with modified myelin self-antigen prevents complete paralysis while avoiding autoimmune disease. J Clin Invest. 2001;108(4):591-599.

9. Walsh JT, et al. MHCII-independent $\mathrm{CD}^{+} \mathrm{T}$ cells protect injured CNS neurons via IL-4. 2015;125(2):699-714.

10. Steinman L. The discovery of natalizumab, a potent therapeutic for multiple sclerosis. J Cell Biol. 2012;199(3):413-416.

11. Rudick R, et al. Natalizumab: bench to bedside and beyond. JAMA Neurol. 2013;70(2):172-182.

12. Yednock T, et al. Prevention of experimental autoimmune encephalomyelitis by antibodies against $\alpha 4 \beta 1$ integrin. Nature. 1992;356(6364):63-66.

13. Hla T, Brinkmann V. Sphingosine 1-phosphate (S1P): Physiology and the effects of S1P receptor modulation. Neurology. 2011;76(8 suppl 3):S3-S8.

14. Chun J, Brinkmann V. A mechanistically novel, first oral therapy for multiple sclerosis: the development of fingolimod (FTY720, Gilenya). Discov Med. 2011;12(64):213-228.

15. Novartis. Novartis provides update on fingolimod Phase III trial in primary progressive MS (PPMS). Novartis Web site. http://www.novartis.com/ newsroom/media-releases/en/2014/1875463. shtml. Updated December 1, 2014. Accessed December 20, 2014. 